\title{
On the capacity of Rayleigh-fading correlated spectrum sharing networks
}

\author{
Behrooz Makki and Thomas Eriksson
}

\begin{abstract}
Recently, substantial attention has been paid to improve the spectral efficiency of communication setups using different spectrum sharing techniques. This paper aims to study the capacity of Rayleigh-fading spectrum sharing channels in the case where there is correlation between the fading channels. Assuming perfect channel state information, the channel capacity is obtained under both adaptive and nonadaptive unlicensed user power allocation capabilities and the licensed user outage probability and received interference power constraints. Theoretical and simulation results show that (1) there is considerable potential for data transmission of unlicensed users with limited degradation of the licensed users' data transmission efficiency, (2) while the effect of fading channels dependencies is ignorable at low correlation values, the channel capacity is significantly affected as the channels correlation increases, and (3) depending on the fading distributions, the unlicensed user channel capacity is not essentially a decreasing function of the licensed user transmission power in the licensed user outage-limited scenario.
\end{abstract}

\section{Introduction}

Spectrum sharing networks are initiated by the apparent lack of spectrum under the current spectrum management policies. Currently, most of frequency bands useful to wireless communication are under control of primary license holders that have exclusive right to transmit over their spectral bands. This is the point that has created the perception of spectrum shortage, leading to evergrowing complaints about available spectral resources. On the other hand, recent studies, e.g., $[1,2]$, show that at any given time, large portions of the licensed bands remain unused or are under utilized. Therefore, it is expected that the data transmission strategies can be improved by better utilization of the licensed resources. Spectrum sharing network is one of the most promising techniques created for this purpose.

Generally, the goal of a spectrum sharing scheme is to better utilize the radio spectrum by allowing the unlicensed secondary users (SUs) to coexist within the spectral resources of the licensed primary users (PUs). Along with the standard interference channel [3-5], where independent transmitters send independent messages to independent receivers, there are other ways such as

\footnotetext{
*Correspondence: behrooz.makki@chalmers.se

Department of Signals and Systems, Chalmers University of Technology, Gothenburg, Sweden
}

interference-avoiding and simultaneous transmission schemes to exploit the idea of spectrum sharing. The interference-avoiding paradigm [6-8] refers to an approach where the SU transmitter, provided that it can sense the spatial, temporal or spectral gaps of the PU resources, can adjust its transmission parameters to fill these white spaces. Although this scheme can theoretically lead to significant spectral efficiency improvement, it suffers from some practical drawbacks mainly related to imperfect gap detection. Moreover, it is not useful in online applications, as the SU transmission is decided by the PU activation status. In the simultaneous transmission approach, on the other hand, a secondary user can simultaneously coexist with a primary user as long as it works below a certain interference level imposed by the primary user quality-of-service requirements [9-14]. In such methods, the limits on the interference level received at the PU receiver, normally referred to as interference temperature, can be considered to be longterm average or short-term peak constraints.

Considering different levels of channel state information (CSI), a large number of scientific reports can be found that have tackled the spectrum sharing problem in different theoretical aspects. For instance, in [8] and [15], the authors presented some information on theoretical models, limits and open problems of spectrum

\section{黑 Springer}

(C) 2011 Makki and Eriksson; licensee Springer. This is an Open Access article distributed under the terms of the Creative Commons Attribution License (http://creativecommons.org/licenses/by/2.0), which permits unrestricted use, distribution, and reproduction in any medium, provided the original work is properly cited. 
sharing networks. Then, assuming perfect SU-PU CSI, Kang et al. [16] investigated the effect of optimal power allocation on the capacity $[7,11,16-19]$ of secondary channel under different power constraints. Kang et al. [20] studied the secondary user channel capacity under different primary user outage constraints. Moreover, Pei et al. [21] investigated the channel data transmission efficiency under primary user received signal-to-noiseand-interference (SINR) constraints. Allowing limited interference power at the PU receiver, Ji et al. [22] analyzed the capacity of multicast spectrum sharing networks. In their work, while the SU-SU link is assumed to be perfectly known, the results are obtained for the cases where the interference information is perfectly or imperfectly available at the SU transmitter. Also, Musavian and Aissa [23] and Musavian and Aissa [24] studied the ergodic, the outage and the minimum-rate capacity of spectrum sharing network under perfect and imperfect SU-PU CSI assumptions. Finally, considering different channel information imperfection model and interference constraints, Suraweera et al. [25] presented the same results as Musavian and Aissa [24] and verified the effect of feedback quantization as well.

References [16,20-25] are all based on the assumption that the fading channels are mutually independent. That is, the channel performance is investigated in the case where there is no correlation between the fading channels of different transmission end-points. However, based on the environmental properties, realistic channels may not be independent [26-30]. Therefore, it is important to study the channel performance under correlated channels condition.

In this perspective, this paper studies the capacity of Rayleigh-fading correlated spectrum sharing networks. Assuming perfect CSI available at the secondary user transmitter and receiver, the channel performance is investigated under different primary user outage probability and instantaneous or average received interference power constraints. The channel capacity is obtained under both adaptive and nonadaptive secondary user power allocation conditions. The results show that there is considerable potential for data transmission of unlicensed users with limited degradation of the licensed users data transmission efficiency. Also, while the effect of fading channels dependencies is ignorable at low correlation values, the channel capacity is significantly affected as the channels correlation increases.

\section{Channel model}

As illustrated in Figure 1, we consider a correlated spectrum sharing network in which two primary and secondary users share the same narrow-band frequency with bandwidth $B$. With no loss of generality, we let $B=$ 1 . We focus on infinite backlogged systems where both users have infinite amount of information for transmission and are always active. Therefore, the channel is

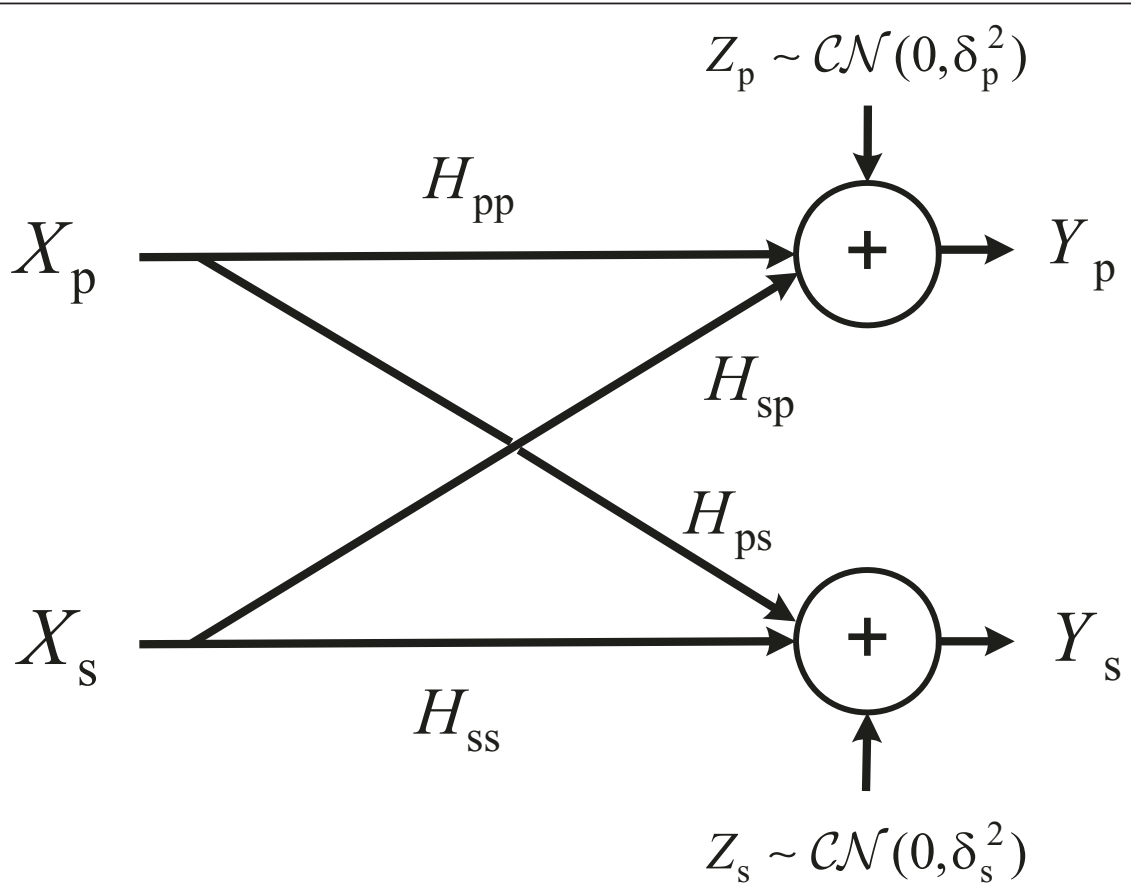

Figure 1 Channel model. Both users share the same narrow-band frequency with bandwidth $B$ and the fading gains are correlated. Section III studies the SU-SU channel capacity under different SU transmission power and the PU received interference power or outage probability constraints. 
modeled as

$$
\left\{\begin{array}{c}
Y_{\mathrm{p}}=X_{\mathrm{p}} H_{\mathrm{pp}}+X_{\mathrm{s}} H_{\mathrm{sp}}+Z_{\mathrm{p}}, Z_{\mathrm{p}} \sim \mathcal{C N}\left(0, \delta_{\mathrm{p}}^{2}\right) \\
Y_{\mathrm{s}}=X_{\mathrm{s}} H_{\mathrm{ss}}+X_{\mathrm{p}} H_{\mathrm{ps}}+Z_{\mathrm{s}}, Z_{\mathrm{s}} \sim \mathcal{C N}\left(0, \delta_{\mathrm{s}}^{2}\right)
\end{array}\right.
$$

in which $X_{\mathrm{s}}$ and $X_{\mathrm{p}}$ represent the power-limited SU and PU input signals, $Y_{\mathrm{s}}$ and $Y_{\mathrm{p}}$ denote their corresponding received signals, and $H_{\mathrm{pp}}, H_{\mathrm{ps}}, H_{\mathrm{ss}}$ and $H_{\mathrm{sp}}$ are the fading random variables in the PU-PU, PU-SU, SU-SU and SU-PU links, respectively. Correspondingly, we define $G_{\mathrm{pp}} \doteq\left|H_{\mathrm{pp}}\right|^{2}, G_{\mathrm{ps}} \doteq\left|H_{\mathrm{ps}}\right|^{2}, G_{\mathrm{sp}} \doteq$ $\left.H_{\mathrm{sp}}\right|^{2}$ and $G_{\mathrm{ss}} \doteq\left|H_{\mathrm{ss}}\right|^{2}$ which are denoted channel gains in the following. Here, we investigate the interference-limited channel performance where, compared to the users received interference, the noise variances are so small that they can be ignored in the calculations. Also, the PU transmission signal is supposed to have Gaussian probability density function (pdf) with fixed power $E\left|X_{\mathrm{p}}\right|^{2}=T_{\mathrm{p}}$. This is a reasonable assumption particularly under perfect CSI condition where Gaussian distributions maximize the PU data transmission efficiency.

We study Rayleigh-fading correlated channels; the channel gains are supposed to have identical exponential pdfs $f_{G}(x)=\lambda e^{-\lambda x}, x \geq 0$, and the relations between every two fading random variable $H_{\mathrm{ij}}$ and $H_{\mathrm{kl}},(\mathrm{i}, \mathrm{j}) \neq(\mathrm{k}, \mathrm{l})$ are modeled by [25]

$$
H_{\mathrm{ij}}=\beta_{\mathrm{ijkl}} H_{\mathrm{kl}}+\sqrt{1-\beta_{\mathrm{ijkl}}^{2}} \varepsilon, \varepsilon \sim \mathcal{C N}\left(0, \frac{1}{\lambda}\right) .
$$

Here, $\beta_{\mathrm{ijkl}}$ is a known correlation factor modeling the two variable dependencies, and $\lambda$ is the exponential pdf parameter determined based on the path loss and shadowing between the terminals. This is a well-established model considered in the literature for different applications [25,31-33]. In this way, the joint pdf of the gains is found as

$$
f_{\mathrm{G}_{\mathrm{ij},}, G_{\mathrm{kl}}}(x, y)=\frac{\lambda^{2}}{1-\beta_{\mathrm{ijkl}}^{2}} e^{-\lambda \frac{x+y}{1-\beta_{\mathrm{ijkl}}^{2}}} I_{0}\left(\frac{2 \beta_{\mathrm{ijkl}} \lambda \sqrt{x y}}{1-\beta_{\mathrm{ijkl}}^{2}}\right)
$$

where $I_{0}($.$) is the zeroth-order modified Bessel func-$ tion of the first kind [34]. It is assumed that there is a perfect channel state information (CSI) available at both transmitters and receivers. Moreover, all results are presented in natural logarithm basis, the simulation results are presented in nats-per-channel-use (npcu), and, as stated in the following, the arguments are restricted to Gaussian input distributions. Finally, we note that Rayleigh-fading channels are good models for tropospheric and ionospheric signal propagation as well as the effect of heavily built-up urban environments on radio signals $[35,36]$. Also, it is most applicable when there is no dominant propagation along a line of sight between the transmitters and the receivers.

\section{Secondary user channel capacity}

With no knowledge about the PU transmitted codeword, the SU-SU channel can be considered as an interference-affected single-input-single-output (SISO) channel. Therefore, the SU-SU channel capacity with perfect channel state information available at the transmitter and receiver can be represented by

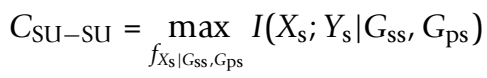

in which $I(U ; V)=h(U)-h(U \mid V)$ denotes the mutual information between two random variables $U$ and $V$, $h(U)=-\int_{-\infty}^{\infty} f_{U}(u) \log \left(f_{U}(u)\right) \mathrm{d} u$ is the differential entropy of the variable $U$ having pdf $f_{U}(u)$, and the maximization is on the secondary user input distribution [19]. On the other hand, representing the SU transmission power by $E\left|X_{\mathrm{s}}\right|^{2}=T_{\mathrm{s}}$, the SU instantaneous received signal-to-interference ratio (SIR) is obtained by

$$
\operatorname{SIR}_{\mathrm{s}}=T_{\mathrm{s}} \Omega_{\mathrm{s}}
$$

in which $\Omega_{\mathrm{s}}$ is an auxiliary random variable defined as $\Omega_{\mathrm{s}} \doteq \frac{G_{\mathrm{ss}}}{T_{\mathrm{p}} G_{\mathrm{ps}}}$. Therefore, due to perfect information about the channel variations, the maximum powerlimited achievable rates of the secondary user are obtained by Gaussian distribution at the transmitter and typical-set-based decoding at the receiver [19], resulting in ${ }^{\mathrm{a}}$

$$
\begin{aligned}
C_{\text {SU-SU }} & =\int_{0}^{\infty} f_{\Omega_{\mathrm{s}}}(\omega) \log \left(1+\omega T_{\mathrm{s}}\right) \mathrm{d} \omega \\
& \stackrel{(a)}{=} \int_{0}^{\infty} \frac{1-F_{\Omega_{\mathrm{s}}}(\omega)}{1+\omega T_{\mathrm{s}}} T_{\mathrm{s}} \mathrm{d} \omega .
\end{aligned}
$$

Here, $f_{\Omega_{\mathrm{s}}}$ and $F_{\Omega_{\mathrm{s}}}$ are the auxiliary variable pdf and cumulative distribution function (cdf), respectively, and (a) is obtained by partial integration and nonnegativity of the variable $\Omega_{\mathrm{s}}$. Therefore, the main problem would be to determine the cdf $F_{\Omega_{\mathrm{s}}}$ and then (6). 
Considering (3), the auxiliary variable cdf is found as

$$
\begin{aligned}
& F_{\Omega_{\mathrm{s}}}(z)=\operatorname{Pr}\left\{\frac{G_{\mathrm{ss}}}{T_{\mathrm{p}} G_{\mathrm{ps}}} \leq z\right\}=\int_{y=0}^{\infty} \int_{x=0}^{x=y z T_{\mathrm{p}}} f_{\mathrm{G}_{\mathrm{ss}}, G_{\mathrm{ps}}}(x, y) \mathrm{d} x \mathrm{~d} y \\
& =\int_{0}^{\infty}\left(\int_{0}^{T_{\mathrm{p}} y z} \frac{\lambda^{2}}{1-\beta_{\mathrm{ssps}}^{2}} e^{-\lambda \frac{x+y}{1-\beta_{\mathrm{ssps}}^{2}}} I_{0}\left(\frac{2 \beta_{\mathrm{ssps}} \lambda \sqrt{x y}}{1-\beta_{\mathrm{ssps}}^{2}}\right) \mathrm{d} x\right) \mathrm{d} y \\
& \stackrel{(n)}{=} \int_{0}^{\infty} \lambda e^{-\lambda \frac{\gamma}{1-\beta_{\mathrm{ssps}}^{2}}} \\
& \sqrt{\frac{2 \lambda}{1-\beta_{\text {ssps }}^{2}} T_{\mathrm{p}} y z} \\
& \times \int_{0}^{\sqrt{1-\beta_{\text {ssps }}^{2}} r T^{-} \gamma z} r e^{-\frac{r^{2}}{2}} I_{0}\left(\beta_{\text {ssps }} \sqrt{\frac{2 \lambda}{1-\beta_{\text {ssps }}^{2}} y r}\right) \mathrm{d} r \mathrm{~d} \gamma \\
& \stackrel{(m)}{=} \int_{0}^{\infty} \lambda e^{-\lambda y}\left\{1-Q\left(\beta_{\mathrm{ssps}} \sqrt{\frac{2 \lambda}{1-\beta_{\mathrm{ssps}}^{2}}} y, \sqrt{\frac{2 \lambda}{1-\beta_{\mathrm{ssps}}^{2}} T_{\mathrm{p}} y z}\right)\right\} \mathrm{d} y \\
& \stackrel{(p)}{=} \frac{1}{2}\left(1+\frac{u}{w}\right) \\
& u=\frac{1}{1-\beta_{\text {ssps }}^{2}}-\frac{1}{\left(1-\beta_{\text {ssps }}^{2}\right) T_{\mathrm{p}} z} \\
& v=\frac{1}{1-\beta_{\text {ssps }}^{2}}+\frac{1}{\left(1-\beta_{\text {ssps }}^{2}\right) T_{\mathrm{p}} z} \\
& w=\sqrt{v^{2}-\frac{4 \beta_{\text {ssps }}^{2}}{\left(1-\beta_{\text {ssps }}^{2}\right)^{2} T_{\mathrm{p}} z}}
\end{aligned}
$$

Here, $(n)$ is obtained by variable transform $x=\frac{1-\beta_{\text {ssps }}^{2}}{2 \lambda} r^{2},(m)$ is based on some calculation, the definition of the Marcum $Q$-function

$$
Q(x, y)=\int_{y}^{\infty} r e^{-\frac{r^{2}+x^{2}}{2}} I_{0}(x r) \mathrm{d} r
$$

and $Q(x, 0)=1$ and $(p)$ is derived using [25, eq. (31)]. Replacing (7) in (6), the power-limited channel capacity is calculated based on the considered power constraints.

\section{A The effect of different PU quality-of-service requirements and SU power allocation capabilities}

Depending on the SU transmitter properties and the PU quality-of-service requirements, there may be different power constraints in a spectrum sharing network. Here, we first study the case where no power allocation is done by the SU transmitter, and the codewords are sent with a fixed power. Later, we will relax this constraint, permitting adaptive power allocation by the $\mathrm{SU}$ transmitter.

\section{PU interference power constraint and nonadaptive SU transmission power}

Limiting the PU average received interference power, which for fixed SU transmission power is found as $T_{\mathrm{s}} E G_{\mathrm{sp}}$, to be less than a threshold $\mu$, we have $T_{\mathrm{s}} \leq \frac{\mu}{E G_{\mathrm{sp}}}$. Then, as the transmission rate of Gaussian channels is an increasing function of the SIR $[37,38]$, the optimal case is obtained by equality. On the other hand, we can instead consider the case where the instantaneous received interference power is with probability $\xi$ less than a threshold $\eta$. In this case, as we have

$$
\operatorname{Pr}\left\{\operatorname{Int}_{\mathrm{p}} \leq \eta\right\}=\operatorname{Pr}\left\{T_{\mathrm{s}} \mathrm{G}_{\mathrm{sp}} \leq \eta\right\}=F_{\mathrm{G}_{\mathrm{sp}}}\left(\frac{\eta}{T_{\mathrm{s}}}\right)
$$

the optimal transmission power satisfying the instantaneous interference power constraint $\operatorname{Pr}\left\{\operatorname{Int}_{\mathrm{p}} \leq \eta\right\} \geq \xi$ is obtained by

$$
T_{\mathrm{s}}=\frac{\eta}{F_{G_{\mathrm{sp}}}^{-1}(\xi)} .
$$

Here, $F_{G_{\mathrm{sp}}}^{-1}($.$) is the inverse function of the SU-PU fad-$ ing cdf which for Rayleigh-fading channels simplifies to $F_{G_{\mathrm{sp}}}^{-1}(x)=\frac{-1}{\lambda} \log (1-x)$.

\section{PU outage probability constraint and nonadaptive SU transmission power}

Assuming that the PU message is transmitted at a fixed rate $R_{\mathrm{p}}$, the PU outage probability ${ }^{\mathrm{b}}$ is found as

$$
\begin{aligned}
\operatorname{Pr} \text { \{ outage }_{\mathrm{p}} & =\operatorname{Pr}\left\{\log \left(1+\frac{T_{\mathrm{p}} G_{\mathrm{pp}}}{T_{\mathrm{s}} \mathrm{G}_{\mathrm{sp}}}\right) \leq R_{\mathrm{p}}\right\} \\
& =\operatorname{Pr}\left\{\frac{G_{\mathrm{pp}}}{T_{\mathrm{s}} G_{\mathrm{sp}}} \leq \frac{e^{R_{\mathrm{p}}}-1}{T_{\mathrm{p}}}\right\}=F_{\Omega_{\mathrm{p}}}\left(\frac{e^{R_{\mathrm{p}}}-1}{T_{\mathrm{p}}}\right)
\end{aligned}
$$

in which $F_{\Omega_{\mathrm{p}}}($.$) is the cdf of the variable \Omega_{\mathrm{p}}=\frac{G_{\mathrm{pp}}}{T_{\mathrm{s}} G_{\mathrm{sp}}}$ obtained with the same procedure as in (7). In this way, constraining the PU outage probability to be less than $\pi_{\mathrm{p}}$, i.e., $\operatorname{Pr}\{\text { outage }\}_{\mathrm{p}} \leq \pi_{\mathrm{p}}$, the SU transmission power is obtained as

$$
\begin{aligned}
& T_{\mathrm{s}}=\left\lceil\frac{T_{\mathrm{p}}}{e^{R_{\mathrm{p}}}-1} \frac{\gamma-\sqrt{\gamma^{2}-\frac{\varphi}{2}}}{\varphi}\right]^{+} \\
& \varphi=2 \pi_{\mathrm{p}}\left(1-\pi_{\mathrm{p}}\right), \\
& \gamma=\varphi+1-\beta_{\mathrm{ppsp}}^{2}
\end{aligned}
$$

where $\lceil x\rceil^{+} \doteq \max \{0, x\}$. Finally, it is worth noting that, with appropriate scalings, (10) can be mapped to the case where, with some probability, the PU received SIR is constrained to be higher than some threshold.

\section{Power adaptation at the SU transmitter}

Intuitively, the SU-SU achievable rates can be increased by adaptive power allocation at the SU transmitter. For instance, we can consider the case where the SU transmission powers are determined such that, while the SU transmission rates are maximized, the PU average received interference power does not exceed a given 
threshold. However, under perfect CSI assumption, the $\mathrm{SU}$ average transmission power is directly related to the PU received interference power. Therefore, we can consider the SU average transmission power instead. In this way, since the average $\mathrm{SU}$ transmission power is obtained by $\bar{T}_{\mathrm{s}}=\int_{0}^{\infty} T_{\mathrm{s}}(\omega) f_{\Omega_{\mathrm{s}}}(\omega) \mathrm{d} \omega$, the optimal transmission power for every SU received SIR realization is found by the Lagrangian objective function

$$
\Upsilon=\int_{0}^{\infty} f_{\Omega_{\mathrm{s}}}(\omega) \log \left(1+\omega T_{\mathrm{s}}(\omega)\right) \mathrm{d} \omega-\rho \int_{0}^{\infty} T_{\mathrm{s}}(\omega) f_{\Omega_{\mathrm{s}}}(\omega) \mathrm{d} \omega(11)
$$

in which $\rho$ is the Lagrange multiplier satisfying the $\mathrm{SU}$ power constraint $\bar{T}_{\mathrm{s}} \leq T$ (or equivalently, the PU average received interference power constraint) [19]. Setting $\frac{\partial \Upsilon}{\partial T_{s}(\omega)}=0$, the optimal transmission powers maximizing (6) are obtained by the water-filling equation

$$
T_{\mathrm{s}}(\omega)=\left\lceil\frac{1}{\rho}-\frac{1}{\omega}\right\rceil^{+}
$$

The Lagrange multiplier $\rho$ is determined based on

$$
\rho=\underset{z}{\arg }\left\{\int_{z}^{\infty}\left(\frac{1}{z}-\frac{1}{\omega}\right) f_{\Omega_{\mathrm{s}}}(\omega) \mathrm{d} \omega=T\right\}
$$

where we have

$$
\begin{aligned}
& \int_{2}^{\infty}\left(\frac{1}{z}-\frac{1}{\omega}\right) f_{\Omega_{1}}(\omega) \mathrm{d} \omega \stackrel{(b)}{=} \int_{2}^{\infty} \frac{1-F_{\Omega_{2}}(\omega)}{\omega^{2}} \mathrm{~d} \omega \\
& \stackrel{(9)}{\underline{g}} T_{\mathrm{p}}\left\{\frac{1}{z T_{\mathrm{p}}}+\frac{1}{2} \sqrt{\left(1+\frac{1}{z T_{\mathrm{p}}}\right)^{2}}-\frac{4 \beta_{\mathrm{sps}}^{2}}{z T_{\mathrm{p}}}\right. \\
& +\left(\beta_{\mathrm{sps}}^{2}-1\right) \log \left(1-2 \beta_{\mathrm{sps}}^{2}+\frac{1}{z T_{\mathrm{p}}}+\sqrt{\left.\left(1+\frac{1}{z T_{\mathrm{p}}}\right)^{2}-\frac{4 \beta_{\mathrm{sps}}^{2}}{z T_{\mathrm{p}}}\right)}\right. \\
& \left.-\frac{1}{2}+\left(1-\beta_{\mathrm{sps} s}^{2}\right) \log \left(2-2 \beta_{\mathrm{sps} s}^{2}\right)\right\}
\end{aligned}
$$

Again, $(b)$ is obtained by partial integration, and $(c)$ is based on (7) and some calculations. Finally, replacing (12) in (6), the SU-SU channel capacity with average SU transmission power (or equivalently, PU average interference power) constraint is obtained by

$$
\begin{aligned}
& C_{\text {sususu }}=\int_{\rho}^{\infty} f_{s_{3}}(\omega) \log \left(1+\omega T_{s}(\omega)\right) \mathrm{d} \omega \\
& =\int_{\rho}^{\infty} f_{\Omega_{3}}(\omega) \log \left(\frac{\omega}{\rho}\right) \mathrm{d} \omega \\
& =\int^{\infty} \frac{1-F_{\Omega_{3}}(\omega)}{\omega} \mathrm{d} \omega \\
& =\frac{1}{2}\left\{\log \left(1-2 \beta_{\mathrm{sps}}^{2}+\frac{1}{\rho T_{\mathrm{P}}}+\sqrt{\left(1+\frac{1}{\rho T_{\mathrm{p}}}\right)^{2}-\frac{4 \beta_{\mathrm{sps}}^{2}}{\rho T_{\mathrm{P}}}}\right)\right. \\
& \left.+\operatorname{arctanh}\left(\frac{1+\frac{1}{\rho T_{\mathrm{p}}}-\frac{2 \beta_{\mathrm{sps}}^{2}}{\rho T_{\mathrm{p}}}}{\sqrt{\left(1+\frac{1}{\rho T_{\mathrm{p}}}\right)^{2}-\frac{4 \beta_{\mathrm{sps}}^{2}}{\rho T_{\mathrm{p}}}}}\right)+\log \left(\frac{1}{\rho T_{\mathrm{p}}}\right)-\log \left(\frac{2-2 \beta_{\mathrm{ssp}}^{2}}{\beta_{\mathrm{sps}}^{2}}\right)\right\}
\end{aligned}
$$

where the second (the third) equality comes from partial integration (partial integration and some manipulations). Finally, it is interesting to note that (12) implies no spectrum sharing at weak channels realizations, i.e., $T_{\mathrm{s}}(\omega)=0, \omega \leq \rho$, where the saved power is spent on good fading realizations, i.e., $T_{\mathrm{s}}(\omega)>0, \omega>\rho$. Then, based on (13), it is easy to show that the threshold $\rho$ is a decreasing function of the average transmission power $T$. That is, more realizations of the variable $\Omega_{\mathrm{s}}$ receive powers as the average transmission power increases (water-filling).

\section{Simulation results}

Assuming fixed SU transmission power and different PU outage probability constraints, Figures 2,3 , and 4 show the SU-SU channel capacity as a function of different parameters. Note that, with proper scalings, the results can be interpreted as the case where, with some probabilities, the PU instantaneous SIR is constrained to be higher than given thresholds. Also, in all simulations, we set $\lambda=1$. Based on (8), the SU-SU channel achievable rates are obtained under PU instantaneous interferencelimited conditions, as illustrated in Figure 5. Here, we set $\beta_{\text {ssps }}=0.3$ and $T_{\mathrm{p}}=1$. Finally, Figure 6 verifies the SU-SU channel capacity under adaptive SU transmission power allocation and limited PU received interference conditions.

\section{Discussions}

Theoretical and simulation results emphasize a number of interesting points that can be listed as follows ${ }^{c}$ :

\section{The effect of PU data transmission in outage-limited conditions}

With a PU outage probability constraint, less rates are achieved at the secondary channel when the PU transmission rate increases (Figure 2a). This is because of the fact that aggressive licensed users require high channel quality. Consequently, they cannot tolerate additive interferences created by the unlicensed users. On the other hand, although no transmission is permitted at low PU transmission powers (Figure $2 \mathrm{~b}$ at low $T_{\mathrm{p}}$ 's), the SU-SU channel capacity increases by the PU power increment in outage-limited conditions (Figure 2b at high $T_{\mathrm{p}}$ 's). However, while this result is valid for Rayleigh-fading channels, it may not be necessarily correct for other pdfs; Under a PU outage probability constraint, the PU transmission power has two different effects on the SU-SU channel capacity. The higher the PU transmission power is, the more relaxed the PU outage probability constraint, i.e., (9), is. This leads to higher transmission powers at the SU transmitter. On the other 


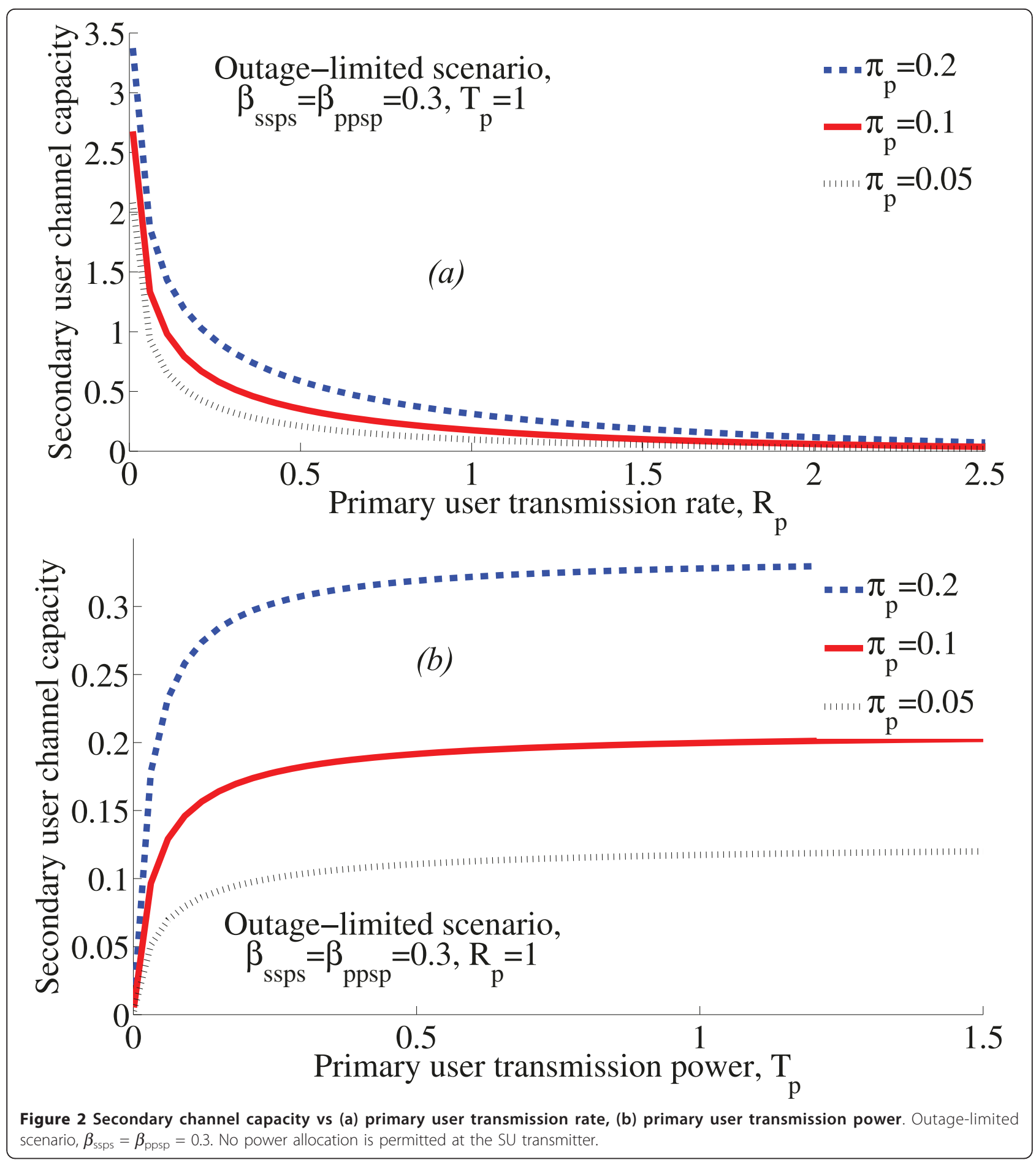

hand, the SU received interference increases by the increment of the PU transmission power which deteriorates the SU-SU channel capacity. Therefore, depending on the fading pdfs, the SU-SU channel capacity is not essentially a monotonic function of the PU transmission power in the PU outage-limited scenario, and the curves may look different from the ones in Figure $2 b$.
The effect of channels correlations

Under both PU outage probability and average received interference power constraints, the SU-SU channel data transmission efficiency is (almost) insensitive to small fading channels correlations (Figures 3, 6c at low correlation values). However, the fading gain dependencies play an important role on the performance of the SU- 

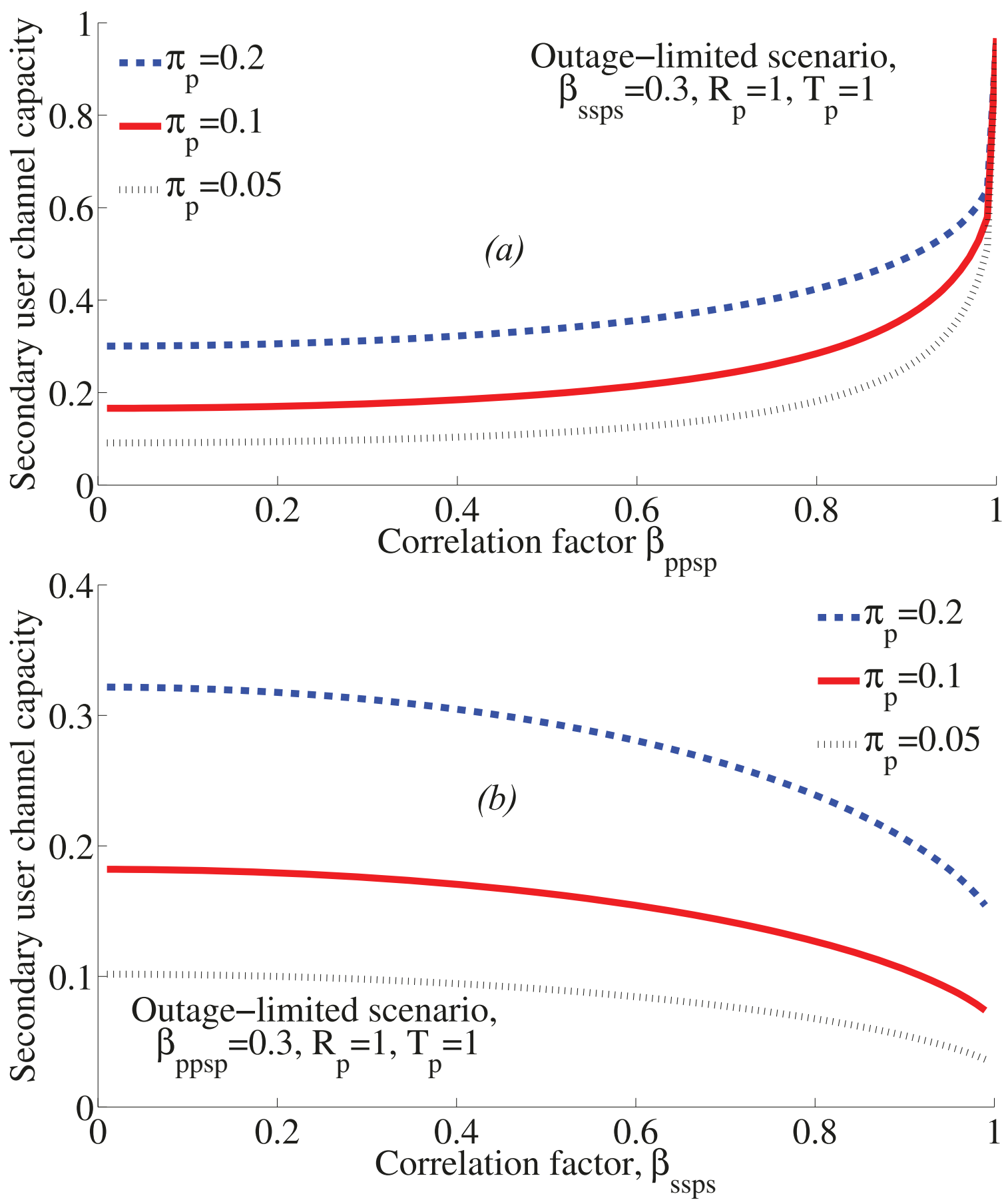

Figure 3 Secondary channel capacity vs the correlations of the (a) PU-PU and PU-SU channels and (b) SU-SU and PU-SU channels Outage-limited scenario, $R_{\mathrm{p}}=1, T_{\mathrm{p}}=1$. No power allocation is permitted at the SU transmitter.

SU channel as the channels correlation increases. Particularly, considering both the outage- and the interference-limited scenarios, the more correlated the SU-SU and PU-SU channels are, the less successful transmission rates are possible for the secondary channel (Figures $3 b, 6 c$ at high correlation values). Considering the auxiliary variable $\Omega_{\mathrm{s}}=\frac{G_{\mathrm{ss}}}{T_{\mathrm{p}} G_{\mathrm{ps}}}$, this is a reasonable result, as under highly correlated conditions both channels change in the same way; Whenever the SU-SU channel is good (bad), the PU-SU channel also experiences high (low) gain realizations. Therefore, the secondary user 


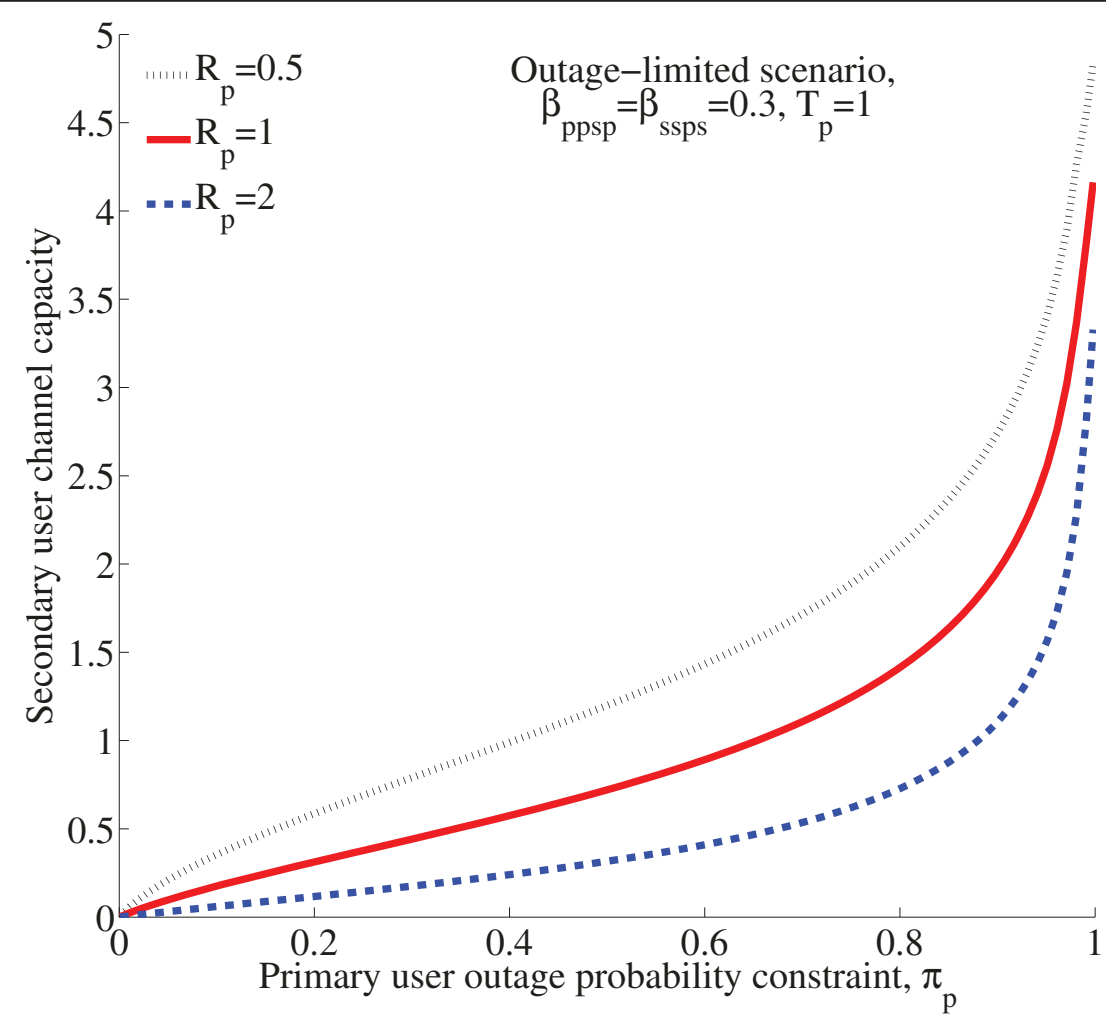

Figure 4 Secondary channel capacity vs primary user outage probability constraint. Outage-limited scenario, $T_{\mathrm{p}}=1, \boldsymbol{\beta}_{\mathrm{ssps}}=\boldsymbol{\beta}_{\mathrm{ppsp}}=0.3$. No power allocation is permitted at the SU transmitter.

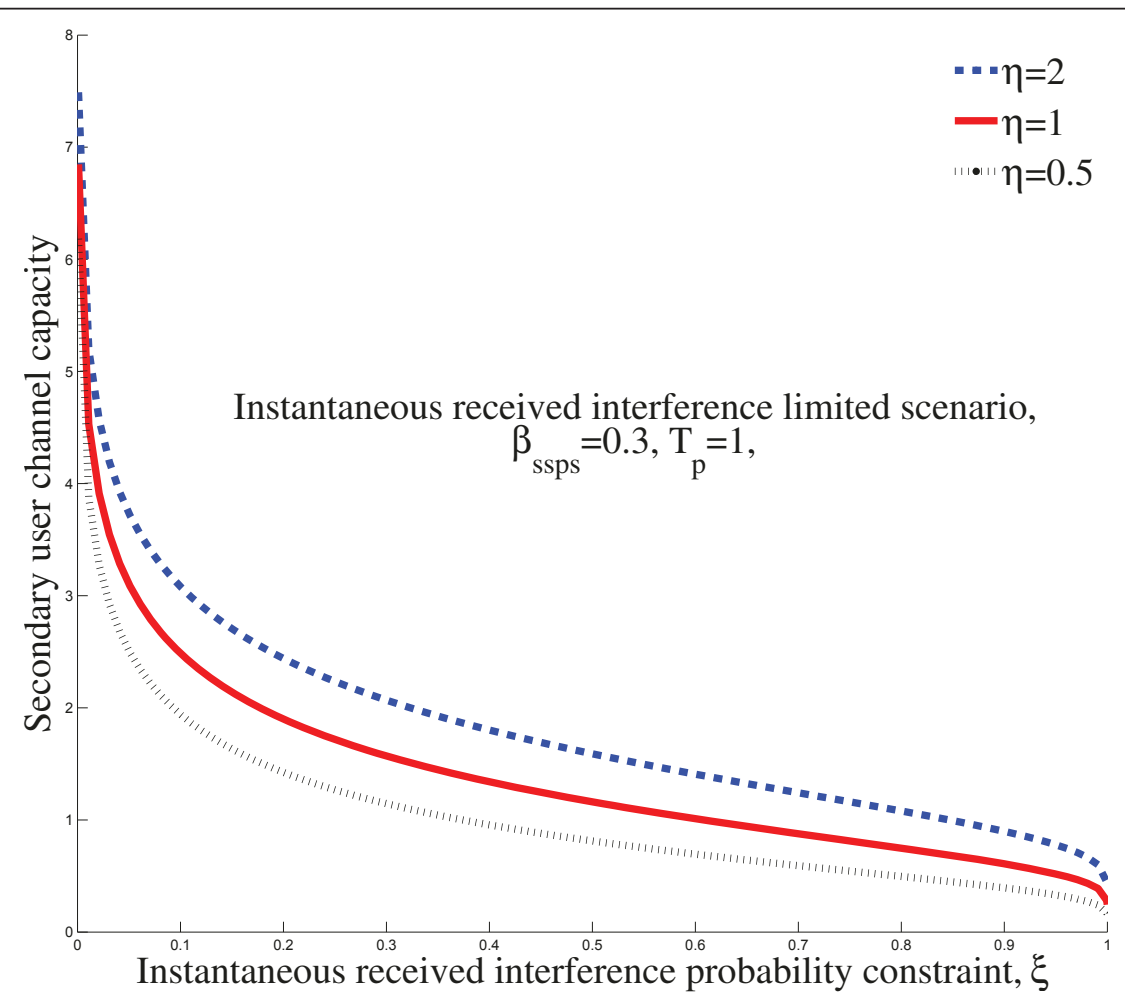

Figure 5 Secondary channel capacity vs instantaneous received interference probability constraint, $\boldsymbol{\xi}$. Interference-limited scenario, $\beta_{\text {ssps }}$ $=0.3, T_{p}=1$. No power allocation is permitted at the $\mathrm{SU}$ transmitter. 

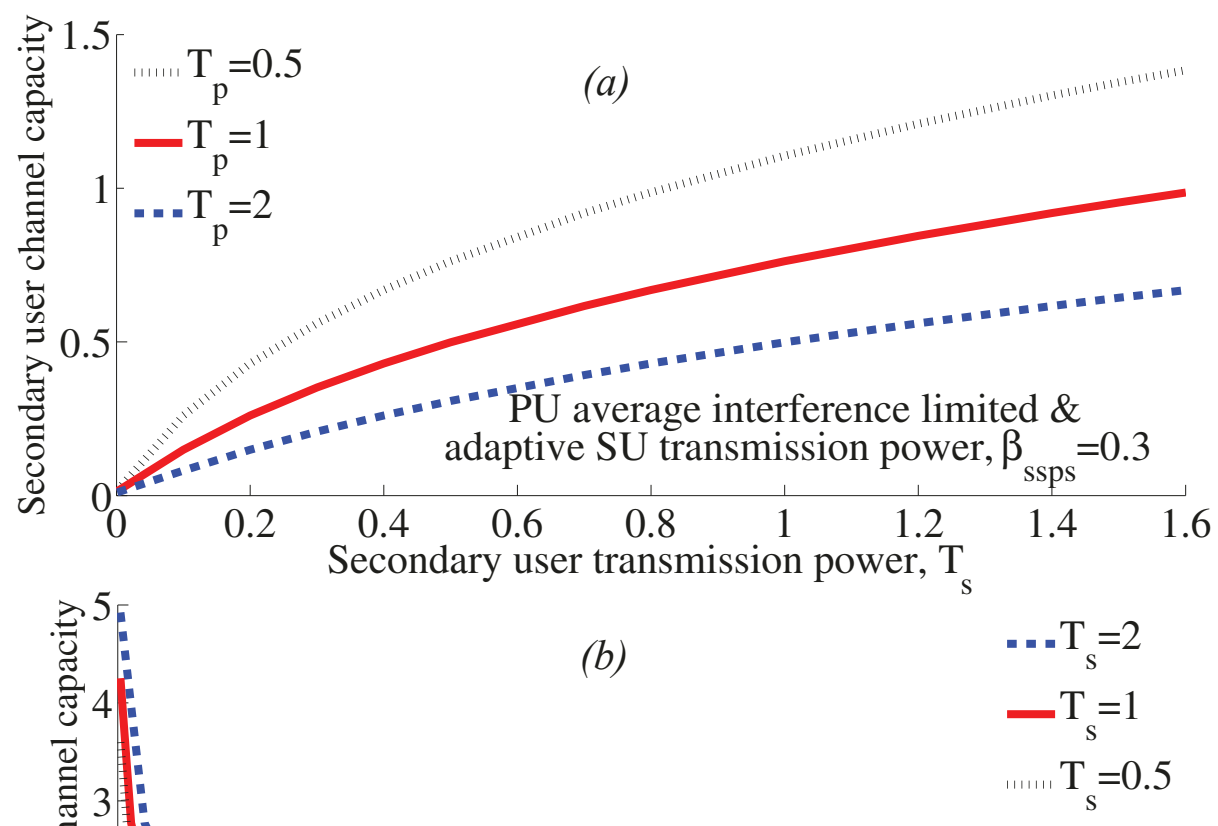

PU average interference limited \&
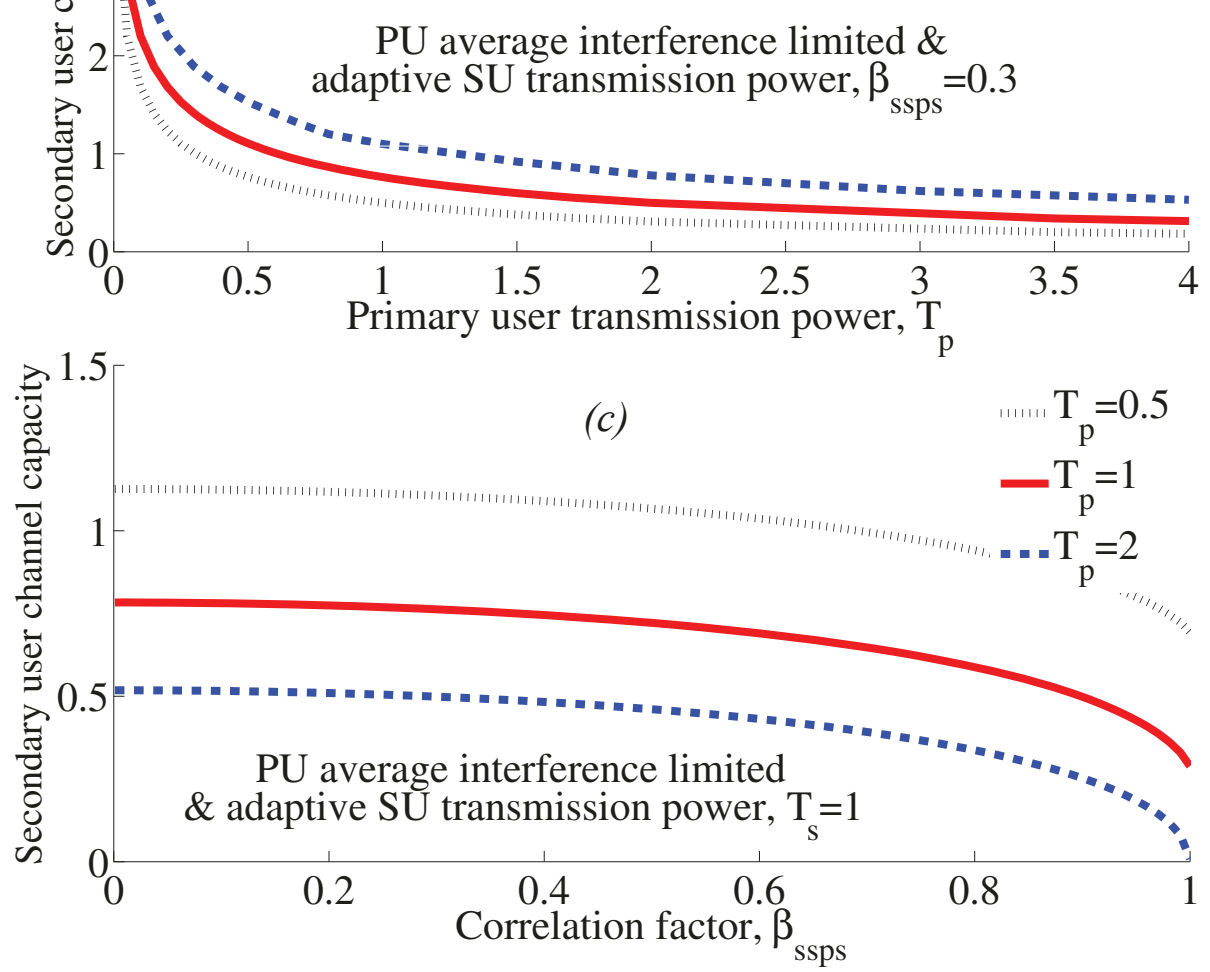

Figure 6 Secondary channel capacity vs (a) SU transmission power, (b) PU transmission power and (c) correlation factor $\boldsymbol{\beta}_{\text {ssps }}$. Adaptive SU transmission power and limited PU average received interference power scenario.

received SIR (and consequently, the SU-SU channel capacity) will be limited. On the other hand, increasing the dependency of the PU-PU and SU-PU channels leads to considerable rate increment in the secondary channel under an outage-limited condition (Figure 3a at high correlation values).

\section{PU tolerability}

The achievable rates of the SU-SU channel are very sensitive to the PU tolerability. For instance, the more the PU instantaneous received interference probability constraint $\xi$ (or the less the PU outage probability constraint $\pi_{\mathrm{p}}$ ) is, the less transmission rates are achieved in the SU- 
SU channel (Figures 4, 5). Particularly, as the probability constraint $\xi$ increases (or the outage probability constraint $\pi_{\mathrm{p}}$ decreases), which means that the PU tolerability decreases, the SU-SU channel capacity converges to zero. That is, no spectrum sharing is permitted.

\section{The effect of PU received interference power constraint}

Considering a PU received interference power constraint, there is considerable potential for data transmission at the secondary channel under both adaptive and nonadaptive SU power allocation conditions (Figures 5, 6). However, the SU-SU channel capacity reduces drastically as the PU transmission power increases (Figure 6b).

\section{Conclusion}

This paper studies the performance of Rayleigh-fading correlated spectrum sharing networks. Considering perfect CSI available at the transmitters and receivers, the unlicensed user channel capacity is investigated under different unlicensed user power allocation capabilities and the licensed user outage probability and (instantaneous or average) received interference power constraints. Simulation results show that, while the effect of channels dependencies is ignorable at low correlation values, the channel capacity is significantly affected as the fading channels correlation increases. Also, it is shown that there is considerable potential for data transmission of unlicensed users even under hard licensed users quality-of-service requirements. Finally, studying the network sum capacity in correlated conditions is left for the future.

\section{Endnotes}

${ }^{a}$ As we know, in an AWGN channel with SIR $x$, the maximum achievable rate is obtained by $\log (1+x)$ [39-41]. ${ }^{\mathrm{b}}$ Note that, with perfect CSI available at the PU receiver, it knows whether the transmitted signal is decodable or not. Therefore, the unreliable messages are disregarded by the receiver and the rest are decoded with no error. Consequently, the outage probability introduced here is not the typical bit or symbol error probability condition but is an average service outage constraint guaranteing the PU data reception at certain percentages of the time slots. For further studies about different service outage constraints, the readers are referred to, e.g., $[42,43]$. ${ }^{\mathrm{c}}$ Note that, as mentioned before, all conclusions derived for PU outage probability constraint can also be used for PU received SIR constraints $\operatorname{Pr}\left\{\operatorname{SIR}_{\mathrm{p}}>\gamma_{\}} \geq \varepsilon\right.$ in which the $\mathrm{PU}$ received SIR is constrained to be higher than a value $\gamma$ with probability of $\varepsilon$.

\section{Competing interests}

The authors declare that they have no competing interests.
Received: 30 March 2011 Accepted: 31 August 2011

Published: 31 August 2011

\section{References}

1. F Berggren, O Queseth, J Zander, B Asp, C Jönsson, NZ Kviselius, B Thorngren, U Landmark, J Wessel, Dynamic Spectrum Access, Phase 1: Scenarios and Research Challenges (2004), http://www.queseth.se/olav/pubs/ DSAReportPhase1.pdf

2. WD Horne, Adaptive Spectrum Access: Using the Full Spectrum Space (2003), http://intel.si.umich.edu/tprc/papers/2003/225/Adaptive_Spectrum_Horne. pdf

3. RH Etkin, DNC Tse, H Wang, Gaussian interference channel capacity to within one bit. IEEE Trans Inf Theory 54(12), 5534-5562 (2008)

4. X Shang, G Kramer, B Chen, A new outer bound and the noisy-interference sum-rate capacity for Gaussian interference channels. IEEE Trans Inf Theory 55(2), 689-699 (2009)

5. D Tuninetti, An outer bound region for interference channels with generalized feedback. Inf Theory Appl Workshop 1, 1-5 (2010)

6. SM Mishra, A Sahai, RW Brodersen, Cooperative sensing among cognitive radios. IEEE Int Conf Commun. 4, 1658-1663 (2006)

7. SA Jafar, S Srinivasa, Capacity limits of cognitive radio with distributed and dynamic spectral activity. IEEE J Sel Areas Commun. 25(3), 529-537 (2007)

8. N Devroye, M Vu, $V$ Tarokh, Cognitive radio networks. IEEE Signal Process Mag. 25(6), 12-23 (2008)

9. B Makki, T Eriksson, Interference management using one bit feedback. Eur Wirel. 1, 1-5 (2011)

10. B Makki, T Eriksson, Capacity bounds of spectrum sharing networks with no channel state information. Eur Wirel. 1, 1-5 (2011)

11. M Gastpar, On capacity under receive and spatial spectrum-sharing constraints. IEEE Trans Inf Theory 53(2), 471-487 (2007)

12. Y Xing, CN Mathur, MA Haleem, R Chandramouli, KP Subbalakshmi, Dynamic spectrum access with QoS and interference temperature constraints. IEEE Trans Mobile Comput. 6(4), 423-433 (2007)

13. W Su, S Lee, DA Pados, JD Matyjas, The optimal transmission power per round for hybrid-ARQ rayleigh fading links. IEEE Int Conf Commun. 1, 1-5 (2010)

14. RA Tannious, A Nosratinia, Cognitive radio protocols based on exploiting hybrid ARQ retransmissions. IEEE Trans Wirel Commun. 9(9), 2833-2841 (2010)

15. N Devroye, $\mathrm{P}$ Mitran, $\mathrm{V}$ Tarokh, Limits on communications in a cognitive radio channel. IEEE Commun Mag. 44(6), 44-49 (2006)

16. X Kang, YC Liang, A Nallanathan, HK Garg, R Zhang, Optimal power allocation for fading channels in cognitive radio networks: Ergodic capacity and outage capacity. IEEE Trans Wirel Commun. 8(2), 940-950 (2009)

17. $F$ Lazarakis, GS Tombras, K Dangakis, Average channel capacity in a mobile radio environment with rician statistics. IECE Trans Commun. E77-B, 971-977 (1994)

18. SM Haas, JH Shapiro, Capacity of wireless optical communications. IEEE J Sel Areas Commun. 21(8), 1346-1357 (2003). doi:10.1109/JSAC.2003.816618

19. TM Cover, JA Thomas, Elements of Information Theory (Wiley, New York, 1992)

20. X Kang, R Zhang, YC Liang, HK Garg, Optimal power allocation strategies for fading cognitive radio channels with primary user outage constraint. IEEE J Sel Areas Commun. 29(2), 374-383 (2011)

21. E Pei, S Wang, Z Zhang, Capacity and optimal power allocation for spectrum-sharing with primary transmission consideration in fading channels. IEEE Commun Lett. 15(4), 389-391 (2011)

22. J Ji, W Chen, H Wan, Y Liu, Capacity analysis of multicast network in spectrum sharing systems. IEEE Int Conf Commun. 1, 1-5 (2010)

23. L Musavian, S Aissa, Capacity and power allocation for spectrum-sharing communications in fading channels. IEEE Trans Wirel Commun. 8(1), 148-156 (2009)

24. L Musavian, S Aissa, Fundamental capacity limits of cognitive radio in fading environments with imperfect channel information. IEEE Trans Commun. 57(11), 3472-3480 (2009)

25. HA Suraweera, PJ Smith, M Shafi, Capacity limits and performance analysis of cognitive radio with imperfect channel knowledge. IEEE Trans Veh Technol. 59(4), 1811-1822 (2010)

26. T Eriksson, T Ottosson, Compression of feedback for adaptive transmission and scheduling. Proc IEEE. 95(12), 2314-2321 (2007) 
27. T Eriksson, T Ottosson, Compression of feedback in adaptive OFDM-based systems using scheduling. IEEE Commun Lett. 11(11), 859-861 (2007)

28. K Huang, R Heath, J Andrews, Limited feedback beamforming over temporally-correlated channels. IEEE Trans Signal Process. 57(5), 1959-1975 (2009)

29. R1-051334, CQl Feedback Scheme for EUTRA, Motorola (RAN1 meeting 43, Seoul, Republic of Korea (2005)

30. R1-062772, Compressed CQI reporting scheme (NEC, RAN WG1 meeting 46, Seoul, Republic of Korea (2006)

31. KS Ahn, RW Heath, Performance analysis of maximum ratio combining with imperfect channel estimation in the presence of cochannel interferences. IEEE Trans Wirel Commun. 8(3), 1080-1085 (2009)

32. Q Sun, DC Cox, HC Huang, A Lozano, Estimation of continuous at fading MIMO channels. IEEE Trans Wirel Commun. 1(4), 549-553 (2002). doi:10.1109/TWC.2002.804178

33. TL Marzetta, BLAST training: Estimating channel characteristics for high capacity space-time wireless, in Proceedings of the 37th Annual Allerton Conference on Communication, Control and Computing. 1(4), 958-966 (1999)

34. C Tellambura, ADS Jayalath, Generation of bivariate rayleigh and nakagamim fading envelopes. IEEE Commun Lett. 4(5), 170-172 (2000). doi:10.1109/ 4234.846501

35. JG Proakis, Digital Communications, 4th edn. (McGraw Hill, New York, 2001)

36. B Sklar, Rayleigh fading channels in mobile digital communication systems.l. characterization. IEEE Commun Mag. 35(7), 90-100 (1997). doi:10.1109/ 35.601747

37. A Lozano, AM Tulino, S Verdu, Optimum power allocation for parallel Gaussian channels with arbitrary input distributions. IEEE Trans Inf Theory 52(7), 3033-3051 (2006)

38. D Guo, S Shamai, S Verdu, Mutual information and minimum mean-square error in Gaussian channels. IEEE Trans Inf Theory. 51(4), 1261-1282 (2005). doi:10.1109/TIT.2005.844072

39. B Makki, T Eriksson, On the average rate of quasi-static fading channels with ARQ and CSI feedback. IEEE Commun Lett. 14(9), 806-808 (2010)

40. B Makki, L Beygi, T Eriksson, Channel capacity bounds in the presence of quantized channel state information. Eura J Wirel Commun Netw. 2010, article ID 495014 (2010)

41. TT Kim, M Skoglund, On the expected rate of slowly fading channels with quantized side information. IEEE Trans Commun. 55(4), 820-829 (2007)

42. K Chakraborty, S Dey, M Franceschetti, Service-outage-based power and rate control for poisson fading channels. IEEE Trans Inf Theory 55(5), 2304-2318 (2009)

43. J Luo, R Yates, P Spasojevic, Service outage based power and rate allocation for parallel fading channels. IEEE Trans Inf Theory 51(7), 2594-2611 (2005). doi:10.1109/TIT.2005.850099

doi:10.1186/1687-1499-2011-83

Cite this article as: Makki and Eriksson: On the capacity of Rayleighfading correlated spectrum sharing networks. EURASIP Journal on Wireless Communications and Networking 2011 2011:83.

\section{Submit your manuscript to a SpringerOpen ${ }^{\mathcal{O}}$ journal and benefit from:}

- Convenient online submission

- Rigorous peer review

- Immediate publication on acceptance

- Open access: articles freely available online

- High visibility within the field

- Retaining the copyright to your article

Submit your next manuscript at $\gg$ springeropen.com 